\title{
Minimal Turn Restrictions for Designing Deadlock-Free Adaptive Routing
}

\author{
Chiu-Chuan Lin and Ferng-Ching Lin \\ Department of Computer Science and Information Engineering \\ National Taiwan University, Taipei, Taiwan, R.O.C.
}

\begin{abstract}
$A$ routing algorithm is basically required to be connected and deadlock-free. We can restrict some directions that messages can turn in a network to avoid deadlock. A deadlock-free adaptive routing with fewer turn restrictions is considered to possess a greater degree of adaptiveness. We present two basic strategies for designing feasible routings on networks which have bidirectional channels. Primer investigation of our strategies reveals their ability to obtain minimal turn restrictions on some typical multicomputer networks, like hypercube, mesh and torus.
\end{abstract}

\section{1: Introduction}

Multicomputers [1] rely on an interconnection network between processors to support the message-passing mechanism. Store-and-forward switching is used in first generation multicomputers. In order to decrease the amount of time spent in transmitting data, wormhole switching $[4,20]$ has been adopted in advanced multicomputer systems, e.g., Ametek 2010, Ncube-2, Intel Paragon, J-machine and iWARP [18].

With wormhole switching, a message is serialized into flit (flow control digit). The header flit of a message contains all the information needed to decide the selection of next channel on the route. As the header flit advances, the subsequent flits follow it in a pipelined fashion. If the header flit encounters a channel already in use, it and its trailing flits are blocked until the channel is freed.

A feasible routing algorithm is required to be connected and deadlock-free. A routing algorithm is connected if it produces at least one routing path for each pair of source and destination nodes. In wormhole routing, deadlock happens when the header flit of a message is destined for a channel which is occupied by another message whose header flit in turn waits for a channel occupied by another message, and so on, and this forms a circular wait situation.
Due to the pipelining transmission of wormhole switching, oblivious routing suffers serious communication performance degradation under heavy or uneven traffic load $[2,3,17,20]$. There are usually many paths between two nodes in a multicomputer. Dynamically choosing a routing path to avoid channel contention may improve the communication latency significantly. Hence, prospective multicomputers are expected to incorporate adaptive routing protocol to diffuse local congestion as well as to provide fault resilience.

Virtual channel is a technique to aid designing deadlock-free wormhole routing algorithms $[5,7,9,14,15]$. However, virtual channel flow control [6] demands extra hardware cost and reduces switching speed. We may employ the turn restriction technique to avoid deadlock without using virtual channels $[10,12]$. In this technique, directions that messages can turn in the network are restricted so that catastrophic deadlock can not arise. Directions that are not restricted provide the zone of adaptiveness that can be flexibly used by the routings.

Under wormhole switching, routing from channel $c_{1}$ to channel $c_{2}$ implies a channel dependency from $c_{1}$ to $c_{2}$. Each cycle in the network indicates a potential channel dependency loop which must be broken to avoid deadlock. Therefore, in each cycle, at least one turn restriction which is formed by a pair of adjacent channels must be restricted to guarantee the deadlock-free property. Clearly, the fewer restrictions are imposed, the more flexibility the messages can be routed with. A routing with minimal turn restrictions is most desirable.

In [10], Glass and Ni presented a turn model. Based on analyzing all the turns in a network and all the cycles that the turns can form, they identified some abstract cycles whose break is just adequate. They applied the turn model to $n$-dimensional mesh networks. All turns in a mesh structure are 90 -degree or 180-degree. The abstract cycles are easy to identify and they found minimal turn restrictions to break all channel dependency cycles in such networks. For $n$-dimensional torus networks, the routings they derived impose sets of turn restrictions that 
are not minimal because the 180-degree turns are considered afterwards. The difficulty encountered by conducting turn model analysis to other networks lies at how to identify all possible abstract cycles and how to prohibit as few turns as possible. In [12], $\mathrm{Li}$ obtained a minimal set of turn restrictions on hypercube by a different reasoning process.

In this paper, we propose a generic scheme to design connected and deadlock-free routings approaching minimal turn restrictions. Our method is applicable to general networks which have bidirectional channels. We present two basic strategies, Strategy $P$ and Strategy $S$, to obtain a set of turn restrictions. Primer investigation reveals that the strategies have the ability to find routings with minimal turn restrictions on some typical multicomputer networks.

\section{2: Preliminaries}

A multicomputer network can be denoted as a digraph $G=(V, C)$, where $V$ is the set of nodes and $C$ is the set of unidirectional channels. The unidirectional channel connecting node $u$ to node $v$ is denoted by $\langle u, v\rangle$. Since we are considering networks that have bidirectional channels, it is required that $\langle u, v\rangle \in C$ if and only if $\langle v, u\rangle \in C$. Also, we assume that there is no self-loop channel, i.e., $\langle u, u>\notin C$ for all $u \in V$.

Define a turn to be an ordered triple $[a, b, c]$, where $<a, b>\epsilon C,\langle b, c>\in C$ and $a \neq c$. Actually, a turn represents a possible dependency in the channel utilization. A turn $[a, b, c]$ is counted as a turn restriction if messages are not allowed to travel from channel $\langle a, b\rangle$ to channel $\langle b, c\rangle$ in the routing.

A distributed controlled adaptive routing algorithm consists of two parts: adaptive set function and output selection policy. The adaptive set function $R: V \times V \times I$ $\rightarrow 2^{C}$, where $I$ is a set of routing informations, determines the set of channels adjacent to the current node that can be chosen to route the message towards the destination. The determined set of channels $R(u, v, i)$ is called the adaptive set associated with the current node $u$, the destination node $v$ and the routing information $i$ the message retains. The output selection policy is a discipline to select a free channel from the adaptive set to route the message.

The routing information can be attached flexibly to produce various routing strategies. In the turn restriction technique, it is required to remember the channel which brings the message to the current node so as to determine the next legitimate channels without violating the turn restrictions. The adaptive set may not contain all possible channels implied by legal turns, this evidently sacrifices some degree of adaptiveness.
The decomposition of adaptive routing into adaptive set function and output selection policy is critical. Different adaptive set functions indicate different degrees of adaptiveness of routing and they also decide whether the algorithm is deadlock-free or not. The output selection policy effects the communication performance under different traffic distributions.

\section{3: Lower bounds of turn restrictions}

From the view point of turn, a directed cycle composed of $n$ processors has $n$ turns. We say a set of directed cycles in a network is turn-disjoint if each pair of cycles in the set have no turn in common. Since imposing at least one turn restriction on each directed cycle is obligatory, the cardinality of a turn-disjoint cycles set gives a lower bound of turn restrictions for a network. The larger size of turn-disjoint cycles set we have, the greater lower bound we obtain. Since the total number of turns in a network is fixed, shorter cycles are considered preferable at finding a larger size of turn-disjoint cycles set.

In the popular $n$-dimensional hypercube $Q_{n}$, there are totally $n(n-1) 2^{n}$ turns. We can find a set of $n(n-1) 2^{n-2}$ turn-disjoint cycles of length 4 . To concretize, each twodimensional plane in the hypercube contains two directed cycles of length 4 having opposite directions and there are $\left(\begin{array}{l}n \\ 2\end{array}\right) 2^{n-2}$ two-dimensional planes in $Q_{n}$. Although hypercube is a subclass of mesh and torus, we explicitly discuss it above to manifest its particular simple formulation.

In a mesh $M_{k_{1} \times k_{2} \times \cdots \times k_{n}}$, where $k_{i}(\geq 2)$ is the size of the $i$-th dimension, we are able to find a set of $2 \sum_{i \neq j}\left[\left(k_{i}-1\right)\left(k_{j}-1\right) \prod_{\substack{p \neq t \\ p \neq j}} k_{p}\right]$ turn-disjoint cycles of length 4 . To see this, each two-dimensional plane formed by the $i$-th and the $j$-th dimensions contains $2\left(k_{i}-1\right)\left(k_{j}-1\right)$ turndisjoint cycles of length 4 and there are $\prod_{\substack{p \neq i \\ p \neq j}} k_{p}$ twodimensional planes formed by the $i$-th and $j$-th dimensions.

In a torus $T_{k_{1} \times k_{2} \times \cdots \times k_{n}}$, where $k_{i}(\geq 3)$ is the size of the $i$-th dimension, we are able to find a set of $2\left(\begin{array}{l}n \\ 2\end{array}\right) \prod_{i=1}^{n}\left[k_{i}+\right.$ $2 \sum_{i=1}^{n} \prod_{j \neq i} k$, turn-disjoint cycles. Here, the first term $2\left(\begin{array}{l}n \\ 2\end{array}\right) \prod_{i=1}^{n} k_{i}$ is the number of turn-disjoint cycles of length 4 and there are $2 \prod_{j \times i} k_{j}$ turn-disjoint cycles of length $k_{i}$.

\section{4: Strategy based on partial-order form}

We draw the network in a partial-order form, called $P$-form such that (1) each channel in the network is 
directed either upward or downward and (2) there is a unique node which can reach every other node in the network via an upward path. We call this unique node the root of the P-form. Appendix A gives a procedure DRAW-P-FORM to draw a network in a P-form. DRAW-P-FORM also outputs a level value $l(u)$ for each node $u$ in the network, which can be utilized in the routing, if necessary.

Lemma 1. Every connected network with bidirectional channels can be drawn in a P-form.

Proof: In DRAW-P-FORM, a node is marked as visited and drawn at some level only when it has no channel connecting to any node in this level. Nodes of level $i+1$ are drawn at one layer above nodes of level $i$. Hence, all channels are directed either upward or downward. In addition, the node at level 0 is the unique node which can reach every other node in the network via an upward path. $\square$

Note that P-form of a network may not be unique. Fig 1(a) is a simple 6-node network, and Fig. 1(b) and Fig. 1(c) are two possible P-forms for it. Each undirected edge in the figures represents a bidirectional channel.

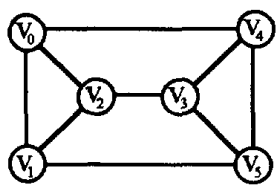

(a) A 6-node network

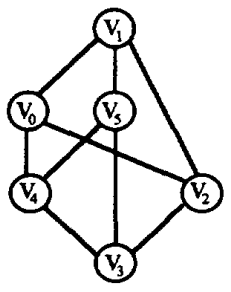

(b) A P-form

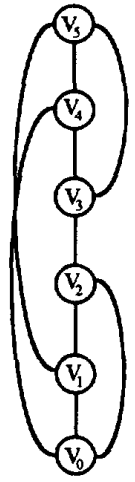

(c) A P-form based on a Hamiltonian path
Fig. 1 An example to illustrate P-forms of a network.

The main idea of Strategy $P$ is as follows. Once a network is drawn in a P-form, each channel is directed either upward or downward. To form a cycle in the network, it requires to make use of some upward channels and some downward channels. Obviously, if we restrict any turn formed by an upward channel and a downward channel (called UD-turn), all cycles are broken. This accommodates the deadlock-free property. Note that if $[a, b, c]$ is a turn restriction then $[c, b, a]$ is also a turn restriction.
Between any pair of nodes, there exists a routing path without UD-turns. For concreteness, assume $u$ is the source node and $v$ is the destination node of a message. If there is no upward path or downward path from $u$ to $v$, the establishment of P-form guarantees the existence of a node $w$ whose level value is less than those of $u$ and $v$, and there is a downward path from $u$ to $w$ and an upward path from $w$ to $v$. Hence, without using the UD-turns, the graph is still connected.

\section{Strategy $\mathbf{P}$}

Step 1. Draw the network in a P-form.

Step 2. Designate all UD-turns as turn restrictions.

Step 3. Define an adaptive set function and an output selection policy. (The adaptive sets must be able to produce at least one routing path for each pair of nodes.)

Theorem 2. Routings produced by Strategy $P$ are connected and deadlock-free.

Strategy $\mathbf{P}$ with different $\mathbf{P}$-forms may designate different number of turn restrictions. If the network is bipartite, the P-form built by procedure DRAW-P-FORM has a particularly simple explanation. It is the same as the breadth-first search spanning tree of the network with the root drawn at the bottom and all other channels in the network added onto the tree. The distance from the root to any node $u$ is equal to $l(u)$. We particularly call a Pform satisfying this condition a $P_{b f s}$-form. In the $\mathrm{P}_{\mathrm{bfs}}{ }^{-}$ form, all channels fall between two adjacent levels.

Networks $Q_{n}, M_{k_{1} \times k_{2} \times \cdots \times k_{n}}$ and $T_{k_{1} \times k_{2} \times \cdots \times k_{n}}$, where $k_{1}, k_{2}$, $\cdots, k_{n}$ are even, are bipartite. By establishing $\mathrm{P}_{\mathrm{bfs}}$-forms for these networks, it happens that the turn restrictions designated by Strategy P exactly reach the lower bounds assessed in the previous section.

Theorem 3. Strategy $P$ with $P_{b f s}$-form designates minimal turn restrictions in $Q_{n}$.

Proof: Since $Q_{n}$ is a vertex symmetric network, we may choose node 0 as the root in the $\mathrm{P}_{\mathrm{bfs}}$-form. Now, a node with $i$ l's in its binary representation is drawn at the $i$-th level. There are $\left(\begin{array}{l}n \\ i\end{array}\right)$ nodes in the $i$-th level. Each node in the $i$-th level has $i$ downward channels to the $(i-1)$-th level and $n-i$ upward channel to the $(i+1)$-th level. Each node $u$ in the $i$-th level endows $\left(\begin{array}{l}i \\ 2\end{array}\right)$ UD-turns of the form $[a, u, c]$. Hence, the total number of UD-turns in the $\mathrm{P}_{\mathrm{bfs}}{ }^{-}$ form is $2\left[\left(\begin{array}{l}n \\ n\end{array}\right)\left(\begin{array}{l}n \\ 2\end{array}\right)+\left(\begin{array}{c}n \\ n-1\end{array}\right)\left(\begin{array}{c}n-1 \\ 2\end{array}\right)+\cdots+\left(\begin{array}{l}n \\ 2\end{array}\right)\left(\begin{array}{l}2 \\ 2\end{array}\right)\right]=n(n-1) 2^{n-2}$. 
Theorem 4. Strategy $P$ with $P_{b f s}$-form designates minimal turn restrictions in $M_{k_{1} \times k_{2} \times \cdots \times k_{n}}$, where $k_{i} \geq 2$.

Proof: The case $n=1$ is trivial so we consider $n \geq 2$. Let $r=\left(r_{1}, r_{2}, \cdots, r_{n}\right)$ be the root in the $\mathrm{P}_{\mathrm{bfs}}$-form. A node $u=\left(u_{1}, u_{2}, \cdots, u_{n}\right)$ has level value $l(u)=\left|n_{1}-u_{1}\right|+\left|r_{2}-u_{2}\right|+$ $\cdots+\left|r_{n}-u_{n}\right|$. We shall calculate the number of UD-turns for each node $u$. Since different two-dimensional planes in $M_{k_{1} \times k_{2} \times \cdots \times k_{n}}$ have no turns in common, we may calculate the number of UD-turns in each two-dimensional plane separately. Let $S M_{k_{\mathrm{p}} \times k_{q}}$ be a two-dimensional plane formed by the $p$-th and the $q$-th dimensions. Node $u$ has at most 4 neighbors in $S M_{k_{p} \times k_{q}}$. Assume $l(u)=i$. There are 3 cases to be discussed.

Case 1. $u_{p}=r_{p}$ and $u_{q}=r_{q}$.

For each neighbor $u^{\prime}$ of node $u, l\left(u^{\prime}\right)=i+1$. There is no UD-turn at $u$ in $S M_{k_{p} \times k_{q}}$.

Case 2. $u_{p}=r_{p}$ and $u_{q} \neq r_{q}$, or $u_{p} \neq r_{p}$ and $u_{q}=r_{q}$.

There is one neighbor $u^{\prime}$ of node $u$ with $l\left(u^{\prime}\right)=i-1$. The other neighbors, if any, have level value $i+1$. There is no UD-turn at $u$ in $S M_{k_{p} \times k_{q}}$

Case 3. $u_{p} \neq r_{p}$ and $u_{q} \neq r_{q}$.

There are two neighbors $u^{\prime}$ and $u^{\prime \prime}$ of $u$ with $l\left(u^{\prime}\right)=l\left(u^{n}\right)=i-1$. The other neighbors, if any, have level value $i+1$. $\left[u^{\prime}, u, u^{\prime \prime}\right]$ and $\left[u^{\prime \prime}, u, u^{\prime}\right]$ are the only UD-turns at $u$ in $S M_{k_{p} \times k_{q}}$

The number of nodes of Case 3 in $S M_{k_{p} \times k_{q}}$ is $\left(k_{p}-1\right)$ $\left(k_{q}-1\right)$. From the above discussions, we know that there are $2\left(k_{p}-1\right)\left(k_{q}-1\right)$ UD-turns in a two-dimensional plane formed by the $p$-th and $q$-th dimensions. Totally, there are $2 \sum_{p \neq q}\left[\left(k_{p}-1\right)\left(k_{q}-1\right) \prod_{\substack{i \neq q \\ i \neq q}} k_{i}\right]$ UD-turns in $M_{k_{1} \times k_{2} \times \cdots \times k_{n}}$

Theorem 5. When $k_{1}, k_{2}, \cdots, k_{n}$ are even and $k_{i} \geq 4$ for $1 \leq i \leq n$, Strategy $P$ with $P_{b f s}$-form designates minimal turn restrictions in $T_{k_{1} \times k_{2} \times \cdots \times k_{n}}$.

Proof: Since $T_{k_{1} \times k_{2} \times \cdots \times k_{n}}$ is a vertex symmetric network, we may consider the root in the $P_{b f s}$-form to be node 0 . The wraparound channels halve the diameter of the corresponding dimension. In the $\mathrm{P}_{\mathrm{bfs}}$-form, we have $l(u)=l\left(u^{\prime}\right)+1$ if $u=\left(u_{1}, u_{2}, \cdots, u_{n}\right)$ and $u^{\prime}=\left(u_{1}^{\prime}, u_{2}^{\prime}, \cdots, u_{n}^{\prime}\right)$ such that $u_{j}=u_{j}^{\prime}$ for all $j \neq i$, and $u_{i}=u_{i}^{\prime}+1$ if $0 \leq u_{i}^{\prime}$ $<\frac{1}{2} k_{i}$ and $u_{i}=u_{i}^{\prime}-1$ if $\frac{1}{2} k_{i}<u_{i}^{\prime} \leq k_{i}$. For convenience, we define $\chi_{i}(a)=a-1$ if $a<\frac{1}{2} k_{i} ; \chi_{i}(a)=a+1 \bmod k_{i}$ otherwise. We shall consider two kinds of turns separately. A turn $[a, b, c]$ is called an xx-turn if both $\langle a, b\rangle$ and $\langle b, c\rangle$ are channels of the same dimension. A turn $[a, b, c]$ is called an xy-turn if channels $\langle a, b\rangle$ and $\langle b, c\rangle$ are of different dimensions. We now calculate the number of UD-turns at node $u=\left(u_{1}\right.$, $\left.u_{2}, \cdots, u_{n}\right)$.

Case 1. xx-turns.

For a node $u=\left(u_{1}, \cdots, u_{i}=\frac{1}{2} k_{i}, \cdots, u_{n}\right),\left[\left(u_{1}, \cdots, u_{i}-1, \cdots\right.\right.$ ,$\left.\left.u_{n}\right), u,\left(u_{1}, \cdots, u_{i}+1, \cdots, u_{n}\right)\right]$ and $\left[\left(u_{1}, \cdots, u_{i}+1, \cdots, u_{n}\right)\right.$, $\left.u,\left(u_{1}, \cdots, u_{i}-1, \cdots, u_{n}\right)\right]$ are two UD-turns that are $\mathrm{xx}-$ turns. There are $\sum_{i=1}^{n} \prod_{j \neq i} k_{j}$ such nodes and all other nodes do not endow any UD-turn which is an xx-turn Hence, there are $2 \sum_{i=1}^{n} \prod_{j \neq i} k_{j}$ UD-turns which are $\mathrm{xx}$ turns.

Case 2. xy-turns.

Consider xy-turns in a two-dimensional plane formed by the $i$-th and the $j$-th dimensions. If $u_{i}=0$ or $u_{j}=0$, node $u$ dose not endow any UD-turn which is an $x y-$ turn. Otherwise, there are 3 subcases to be discussed.

2a. $u_{i} \neq \frac{k_{i}}{2}$ and $u_{j} \neq \frac{k_{j}}{2}$.

Assume $i<j$. We have the UD-turns $\left[\left(u_{1}, \cdots, \chi_{i}\left(u_{i}\right)\right.\right.$ $\left.\left., \cdots, u_{j}, \cdots, u_{n}\right), u,\left(u_{1}, \cdots, u_{i}, \cdots, \chi_{j}\left(u_{j}\right), \cdots, u_{n}\right)\right]$ and [ $\left.\left(u_{1}, \cdots, u_{i}, \cdots, \chi_{j}\left(u_{j}\right), \cdots, u_{n}\right), u,\left(u_{1}, \cdots, \chi_{i}\left(u_{j}\right), \cdots, u_{j}, \cdots, u_{n}\right)\right]$ that are xy-turns. There are $\left(k_{i}-2\right)\left(k_{j}-2\right)$ such nodes endowing $2\left(k_{i}-2\right)\left(k_{j}-2\right)$ UD-turns.

2b. $u_{i}=\frac{k_{i}}{2}$ and $u_{j} \neq \frac{k_{j}}{2}$.

Assume $i<j$. We have the UD-turns $\left[\left(u_{1}, \cdots, u_{i} \pm 1\right.\right.$ $\left.\left., \cdots, u_{j}, \cdots, u_{n}\right), u,\left(u_{1}, \cdots, u_{i}, \cdots, \chi_{j}\left(u_{j}\right), \cdots, u_{n}\right)\right]$ and $\left[\left(u_{1}, \cdots, u_{i}, \cdots, \chi_{j}\left(u_{j}\right), \cdots, u_{n}\right), u,\left(u_{1}, \cdots, u_{i} \pm 1, \cdots, u_{j}, \cdots, u_{n}\right)\right]$ that are $x y$-turns. There are $k_{j}-2$ such nodes endowing $4\left(k_{j}-2\right)$ UD-turns.

2c. $u_{i}=\frac{k_{i}}{2}$ and $u_{j}=\frac{k_{j}}{2}$.

We have the UD-turns $\left[\left(u_{1}, \cdots, u_{i} \pm 1, \cdots, u_{j}, \cdots, u_{n}\right)\right.$, $\left.u, \quad\left(u_{1}, \cdots, u_{i}, \cdots, u_{j} \pm 1, \cdots, u_{n}\right)\right]$ and $\left[\left(u_{1}, \cdots, u_{i}, \cdots\right.\right.$, $\left.\left.u_{j} \pm 1, \cdots, u_{n}\right), u,\left(u_{1}, \cdots, u_{i} \pm 1, \cdots, u_{j}, \cdots, u_{n}\right)\right]$ that are xy-turns. There is only one such node that endows 8 UD-turns.

In summary, there are $2\left(k_{i}-2\right)\left(k_{j}-2\right)+4\left(k_{i}-2\right)+$ $4\left(k_{j}-2\right)+8=2 k_{i} k_{j}$ UD-turns which are xy-turns in a two-dimensional plane formed by the $i$-th and the $j$ th dimensions. Hence, there are totally $\sum_{i \neq j}\left(2 k_{i} k_{j} \prod_{\substack{p \neq i \\ p \neq j}} k_{p}\right)=2\left(\begin{array}{c}n \\ 2\end{array}\right) \prod_{i=1}^{n} k_{i}$ UD-turns which are $\mathrm{xy}-$ turns.

From the above discussions, we conclude that there are $2\left(\begin{array}{l}n \\ 2\end{array}\right) \prod_{i=1}^{n} k_{i}+2 \sum_{i=1}^{n}\left(\prod_{j \neq i} k_{j}\right)$ UD-turns in the $\mathrm{P}_{\mathrm{bfs}}$-form. 
Hamiltonian path of a network provides a special way to draw the network in a P-form [13], for example, see Fig. 1(c). In this P-form, each node has a unique level value in $\{0,1, \cdots,|V|-1\}$, and if $u$ and $v$ are two nodes such that $l(u)=l(v)+1$ then $\langle u, v\rangle \in C$. We call a Pform satisfying this condition a $P_{H p}$-form. Using binaryreflected Gray code (BRGC) sequence in $Q_{n}[19]$ and some special sequence in $M_{k_{1} \times k_{2} \times \cdots \times k_{n}}$ as the Hamiltonian path in the $\mathrm{P}_{\mathrm{Hp}}$-form, the routings based on Strategy $\mathrm{P}$ have minimal turn restrictions.

Let $G(b)=g=g_{n-1} g_{n-2} \cdots g_{0}$ be the BRGC encoding of the binary number $b=b_{n-1} b_{n-2} \cdots b_{0}$. The conversion between binary encoding and BRGC encoding is found to be $G(b)_{n-1}=g_{n-1}=b_{n-1}, G(b)_{i}=g_{i}=b_{i+1} \oplus b_{i}, 0 \leq i \leq n-2 ;$ and $G^{-1}(g)_{i}=b_{i}=g_{n-1} \oplus g_{n-2} \cdots \oplus g_{i}, 0 \leq i \leq n-1$. In network $Q_{n}$, by using BRGC sequence as the Hamiltonian path in the $\mathrm{P}_{\mathrm{Hp}}$-form, the level $l(u)$ of a node $u=u_{n-1} u_{n-2} \cdots u_{0}$ is $l(u)=$ $u_{n-1} 2^{n-1}+\left(u_{n-1} \oplus u_{n-2}\right) 2^{n-2}+\cdots+\left(u_{n-1} \oplus \cdots \oplus u_{1}\right) 2^{1}+\left(u_{n-1} \oplus \cdot \oplus u_{0}\right) 2^{0}$ $=G^{-1}(u)_{n-1} 2^{n-1}+G^{-1}(u)_{n-2} 2^{n-2}+\cdots+G^{-1}(u)_{1} 2^{1}+G^{-1}(u)_{0} 2^{0}$.

Theorem 6. With BRGC sequence as the Hamiltonian path in the $P_{H p}$-form of $Q_{n}$, Strategy $P$ designates minimal turn restrictions.

Proof: We first prove that each undirected cycle of length 4 in $Q_{n}$ is of the form $[a, b, c, d]$ with $l(a)<l(b)<$ $l(c)<l(d)$. Let $u=u_{n-1} u_{n-2} \cdots u_{0}$ and $v=v_{n-1} v_{n-2} \cdots v_{0}$ be two nodes in $Q_{n}$ with Hamming distance equal to 2 . Let $p$ and $q$ be the two bit positions where $u$ and $v$ differ. Assume $p>q$. If $l(u)<l(v)$, then $G^{-1}(u)_{i}=G^{-1}(v)_{i}$ for $p<i \leq n-1, G^{-1}(u)_{p}=0, G^{-1}(v)_{p}=1, G^{-1}(u)_{i}=\overline{G^{-1}(v)_{i}}$ for $q<i<p$ and $G^{-1}(u)_{q}=G^{-1}(v)_{q}$. For convenience, we use $a^{i}$ to denote the neighbor of node $a=\left(a_{n-1}, \cdots, a_{i}\right.$, $\left.\cdots, a_{0}\right)$ across dimension $i$, i.e., $a^{i}=\left(a_{n-1}, \cdots, \bar{a}_{i}, \cdots, a_{0}\right)$. There are two cases to be discussed.

For the first case $G^{-1}(u)_{q}=G^{-1}(v)_{q}=1$, we take $w=u^{p}$ and $w^{\prime}=u^{q}$. Since $G^{-1}(u)_{p}=0, G^{-1}(w)_{p}=G^{-1}\left(u^{p}\right)_{p}=1$ and $G^{-1}(u)_{i}=G^{-1}(w)_{i}$ for $p<i \leq n-1$, we have $l(u)<l(w)$. Also, $G^{-1}(w)_{q}=G^{-1}\left(u^{p}\right)_{q}=0, G^{-1}(v)_{q}=1$ and $G^{-1}(w)_{i}=$ $G^{-1}(v)_{i}$ for $q<i \leq n-1$, we have $l(w)<l(v)$. So, $l(u)<l(w)<l(v)$. In addition, since $G^{-1}\left(w^{\prime}\right)_{q}=G^{-1}\left(u^{q}\right)_{q}$ $=0, G^{-1}(u)_{q}=1$ and $G^{-1}\left(w^{\prime}\right)_{i}=G^{-1}(u)_{j}$ for $q<i \leq n-1$, we have $l\left(w^{\prime}\right)<l(u)$. Therefore, $l\left(w^{\prime}\right)<l(u)<l(w)<l(v)$.

For the second case $G^{-1}(u)_{q}=G^{-1}(v)_{q}=0$, we take $w=u^{q}$ and $w^{\prime}=u^{p}$. Since $G^{-1}(u)_{q}=0, G^{-1}(w)_{q}=G^{-1}\left(u^{q}\right)_{q}=1$ and $G^{-1}(u)_{i}=G^{-1}(w)_{i}$ for $q<i \leq n-1$, we have $l(u)<l(w)$. Also, $G^{-1}(w)_{p}=G^{-1}\left(u^{q}\right)_{p}=0, G^{-1}(v)_{p}=1$ and $G^{-1}(w)_{i}=$
$G^{-1}(v)_{i}$ for $p<i \leq n-1$, we have $l(w)<l(v)$. So, $l(u)<l(w)<l(v)$. In addition, since $G^{-1}(v)_{q}=0$, $G^{-1}\left(w^{\prime}\right)_{q}=G^{-1}\left(u^{p}\right)_{q}=1$ and $G^{-1}(v)_{i}=G^{-1}\left(w^{\prime}\right)_{i}$ for $q<i \leq n-1$, we have $l(v)<l\left(w^{\prime}\right)$. Therefore, $l(u)<l(w)<l(v)<l\left(w^{\prime}\right)$.

The above discussion confirms that there is a UD-turn for each directed cycle of length 4 . From Section 3 , we know that there are totally $n(n-1) 2^{n}$ turns and there are $n(n-1) 2^{n-2}$ directed cycles of length 4 which are turndisjoint. Hence, each turn in $Q_{n}$ belongs to a unique directed cycle of length 4 . So, there are $n(n-1) 2^{n-2}$ UDturns in the $\mathrm{P}_{\mathrm{Hp}}$-form.

Not coincidentally, most suggested multicomputer networks are Hamiltonian. Strategy $P$ with a $\mathrm{P}_{\mathrm{Hp}}$-form has the advantage that the routing can be easily designed. Appendix $\mathrm{C}$ gives an adaptive routing algorithm based on Strategy $\mathrm{P}$ with $\mathrm{P}_{\mathrm{Hp}}$-form. A more detailed examination of applying Strategy $P$ with a $P_{\mathrm{Hp}}$-form to hypercube network can be found in [13]. There we proved that at least one of the shortest paths between any two nodes is contained in the set of routing paths provided by the routing algorithm using BRGC sequence as the Hamiltonian path in the $\mathrm{P}_{\mathrm{Hp}}$-form.

\section{5: Strategy based on breadth-first search}

In the second strategy, Strategy S, we draw a network in an $S$-form as follows. (1) Construct a breadth-first search spanning tree of the network with the root drawn at the bottom. (2) Add all other links in the network onto the spanning tree. Clearly, once the root is fixed, the Sform of a network is unique. Appendix B gives the procedure to draw a network in an S-form. The root of an $\mathrm{S}$-form serves the requisite to design a connected routing algorithm too.

Some channels may lie horizontally between processors in the same level of an S-form of a network. We call a turn that is formed by an upward channel and a horizontal channel a UH-turn, a turn that is formed by a horizontal channel and a downward channel an HD-turn, and a turn that is formed by two consecutive horizontal channels an HH-turn. In contrast to designating all UDturns as turn restrictions to break all cycles in a P-form, we may designate all UD-turns, UH-turns and HH-turns as turn restrictions in the S-form. But, this evidently designates more HH-turns as turn restrictions than needed. We may focus on the connected components of the subgraph induced by the nodes along with all channels in the HH-turns and try to impose sufficient turn restrictions on them. 


\section{Strategy $\mathbf{S}$}

Step 1. Draw the network in an S-form.

Step 2. Designate the following turns as turn restrictions:

(a) UD-turns.

(b) UH-turns.

(c) For each level, consider each connected component $G^{\prime}$ of the subgraph induced by nodes in the level. If $\left|G^{\prime}\right|>1$, then apply Strategy P or Strategy $S$ to obtain a set of turn restrictions on $G^{\prime}$.

Step 3. Define an adaptive set function and an output selection policy. (The adaptive sets must be able to produce at least one routing path for each pair of nodes.)

Theorem 7. Routings produced by Strategy $S$ are connected and deadlock-free.

Note that when the network is bipartite, the S-form is also a $\mathrm{P}_{\mathrm{bfs}}$-form. In Strategy $\mathrm{P}$, a network may have a wide variety of $P$-forms with the same root. In contrast, there is a unique S-form for a network once the root is fixed. There may be different ways to designate turn restrictions in an S-form due to step 2(c) of Strategy S. Also note that, as opposed to Strategy $\mathrm{P}$, when $[a, b, c]$ is a turn restriction, $[c, b, a]$ may not be a turn restriction in Strategy $S$ due to step 2(b).

We have successfully applied Strategy $S$ on $T_{k_{1} \times k_{2} \times \cdots \times k_{n}}$ to designate a set of minimal turn restrictions when $k_{i}$ 's are not all even and $k_{i} \geq 3$ for $1 \leq i \leq n$. We briefly sketch it as follows. $T_{k_{1} \times k_{2} \times \cdots \times k_{n}}$ is a vertex symmetric network, so we can let node 0 be the root in the S-form. We consider xx-turns and xy-turns, as defined in Theorem 5, separately.

Case 1. xx-turns.

If $k_{i}$ is even, following the same discussion in Theorem 5 , there are $2 \prod_{j \neq i} k$, UD-turns formed by the $i$ th dimensional channels. If $k_{i}$ is odd, $\left[\left(u_{1}, \cdots, \frac{k_{i}-3}{2}\right.\right.$, $\left.\left.\cdots, u_{n}\right), \quad\left(u_{1}, \cdots, \frac{k_{i}-1}{2}, \cdots, u_{n}\right), \quad\left(u_{1}, \cdots, \frac{k_{i}+1}{2}, \cdots, u_{n}\right)\right] \quad$ and $\left[\left(u_{1}, \cdots, \frac{k_{i}+3}{2}, \cdots, u_{n}\right),\left(u_{1}, \cdots, \frac{k_{i}+1}{2}, \cdots, u_{n}\right),\left(u_{1}, \cdots, \frac{k_{i}-1}{2}, \cdots, u_{n}\right)\right]$ are two UH-turns. There are $2 \prod_{j \neq i} k_{j}$ UH-turns formed

by the $i$-th dimensional channels. Totally, there are $2 \sum_{i=1}^{n} \prod_{j \neq i} k_{j}$ turns restrictions which are $\mathrm{xx}$-turns.

Case 2. xy-turns.

Consider xy-turns in a two-dimensional plane formed by the $i$-th and the $j$-th dimensions. Let $u=\left(u_{1}, u_{2}, \cdots, u_{n}\right)$ be a node in the two-dimensional plane. If $u_{i}=0$ or $u_{j}=0$, then node $u$ dose not endow either UD-turn or UH-turn or HH-turn formed by the $i$-th and the $j$-th dimensional channels. If $u_{i} \neq 0$ and $u_{j} \neq 0$, we have to consider the parities of $k_{i}$ and $k_{j}$.

2a. Both $k_{i}$ and $k_{j}$ are of even parity.

The discussion is the same as Cases $2 \mathrm{a}-2 \mathrm{c}$ in Theorem 5. There are $2 k_{i} k_{j}$ UD-turns in the twodimensional plane.

$2 \mathrm{~b}$. Both $k_{i}$ and $k_{j}$ are of odd parity.

There are 3 cases. When $u_{i} \neq \frac{k_{i} \pm 1}{2}$ and $u_{j} \neq \frac{k_{j} \pm 1}{2}$, node $u$ endows two UD-turns. There are $\left(k_{i}-3\right)$ $\left(k_{j}-3\right)$ such nodes and they totally endow $2\left(k_{i}-3\right)\left(k_{j}-3\right)$ UD-turns. When $u_{i}=\frac{k_{i} \pm 1}{2}$ and $u_{j} \neq \frac{k_{j} \pm 1}{2}$, node $u$ endows one UH-turn and two UDturns. There are $2\left(k_{j}-3\right)$ such nodes. When $u_{i}=\frac{k_{j} \pm 1}{2}$ and $u_{j}=\frac{k_{j} \pm 1}{2}$, node $u$ endows two UD-turns and two UH-turns. There are 4 such nodes and there are 8 HH-turns among them. In fact, we can designate two HH-turns as turn restrictions to break all cycles formed by the $8 \mathrm{HH}$-turns. In summary, we designate $2\left(k_{i}-3\right)\left(k_{j}-3\right)+6\left(k_{i}-3\right)+6\left(k_{j}-3\right)+4 \times 4+2=2 k_{i} k_{j}$ turn restrictions.

2c. $k_{i}$ is of even parity and $k_{j}$ is of odd parity.

There are 4 cases. When $u_{i} \neq \frac{k_{i}}{2}$ and $u_{j} \neq \frac{k_{j} \pm 1}{2}$, node $u$ endows two UD-turns. There are $\left(k_{i}-2\right)\left(k_{j}-3\right)$ such nodes. When $u_{i} \neq \frac{k_{i}}{2}$ and $u_{j}=\frac{k_{j} \pm 1}{2}$, node $u$ endows one UH-turn and two UD-turns. There are $2\left(k_{i}-2\right)$ such nodes. When $u_{i}=\frac{k_{i}}{2}$ and $u_{j} \neq \frac{k_{j} \pm 1}{2}$, node $u$ endows 4 UD-turns. There are $k_{j}-3$ such nodes. When $u_{i}=\frac{k_{i}}{2}$ and $u_{j}=\frac{k_{j} \pm 1}{2}$, node $u$ endows 4 UD-turns and 2 UH-turns. There are 2 such nodes. In summary, we designate $2\left(k_{i}-2\right)\left(k_{j}-3\right)+$ $6\left(k_{i}-2\right)+4\left(k_{j}-3\right)+2 \times 6=2 k_{i} k_{j}$ turn restrictions.

In conclusion, we designate $2 k_{i} k_{j}$ turn restrictions in a two-dimensional plane formed by $i$-th and $j$-th dimensions, which are irrelevant to the parities of $k_{i}$ and $k_{j}$. Therefore, we totally designate $\sum_{i \neq j}\left(2 k_{i} k_{j} \prod_{\substack{p^{*} \neq j \\ p \neq j}} k_{p}\right)=2\left(\begin{array}{l}n \\ 2\end{array}\right) \prod_{i=1}^{n} k_{i}$ turn restrictions which are xy-turns.

\section{6: Concluding remarks}

In this paper, we have presented two basic strategies to designate a set of turn restrictions on a multicomputer network to design connected and deadlock-free adaptive routing algorithms. The strategies possess the ability to designate minimal turn restrictions on some typical multicomputer networks. 
For $n$-dimensional cube-connected cycles network, the lower bound number of turn restrictions is found to be $n \cdot 2^{n-1}+2^{n+1}$. We are able to establish a specific P-form and an S-form with some modification that produce minimal turn restrictions for $n=3$. We have established modified S-forms with minimal turn restrictions for cube-connected cycles networks up to dimension 5.

We do not dwell upon the details of determining the adaptive set function and output selection policy to complete the design of an adaptive routing algorithm. Network congestion information and length of routing paths are factors contributing to the communication performance of a routing algorithm. If virtual-channel flow control [6] is supported in a network, Duato's theory $[8,9]$ can be incorporated to make use of extra set of virtual channels.

\section{References}

[1] W. C. Athas and C. L. Seitz, "Multicomputers: messagepassing concurrent computers," IEEE Computer, vol. 21, no. 8, pp. 9-24, 1988

[2] S. Chittor and R. Enbody, "Performance evaluation of the mesh-connected wormhole-routed networks for interprocessor communication in multicomputers," Proc. Supercomputing '90, pp. 647-656, 1990.

[3] S. Chittor and R. Enbody, "Performance degradation in large wormhole-routed interprocessor communication networks," Proc. 1990 International Conference on Parallel Processing, vol. I, pp. 424-428, 1990.

[4] W. J. Dally and C. L. Seitz, "The torus routing chip," Jourmal of Distributed Computing, vol. 1, no. 3, pp. 187196, 1986.

[5] W. J. Dally and C. L. Seitz, "Deadlock-free message routing in multiprocessor interconnection networks," IEEE Trans. Computers., vol. 36, no. 5, pp. 547-553, May 1987.

[6] W. J. Dally, "Virtual-channel flow control," IEEE Trans. Parallel and Distributed Systems., vol. 3, no. 2, pp. 194205, March 1992

[7] W. J. Dally and H. Aoki, "Deadlock-free adaptive routing in multicomputer networks using virtual channels," IEEE Trans. Parallel and Distributed Systems, vol. 4, no. 4, pp. 466-475, April 1993.

[8] J. Duato, "Deadlock-free adaptive routing algorithms for multicomputers: evaluation of a new algorithm," Proc. Third IEEE Symp. Parallel and Distributed Processing, pp. 840-847, 1991.

[9] J. Duato, "On the design of deadlock-free adaptive routing algorithms for multicomputers: design methodologies," Proc. Parallel Architectures and Languages Europe 91, pp. 391-405, 1991.

[10] C. J. Glass and L. M. Ni, "The turn model for adaptive routing," Proc. 19th Int'l Symp. Computer Architecture, pp. 278-287, 1992.

[11] I. S. Gopal, "Prevention of store-and-forward deadlock in computer networks," IEEE Trans. Communications, vol. 33 , no. 12, pp. 1258-1264, 1985.
[12] Q. Li, "Minimal deadlock-free message routing restrictions in binary hypercubes," Journal of Parallel and Distributed Computing, vol. 15, no. 2, pp. 153-159, 1992.

[13] C. C. Lin and F. C. Lin, "A general adaptive wormhole routing strategy and its application in hypercube networks," Proc. 1993 Int'l Conf. on Parallel and Distributed Systems (Taipei, Taiwan), pp. 698-703, Dec. 1993.

[14] C. C. Lin and F. C. Lin, "Minimal fully adaptive wormhole routing on hypercubes," to appear in Information Processing Letters.

[15] D. H. Linder and J. C. Harden, "An adaptive and fault tolerant wormhole routing strategy for $k$-ary $n$-cubes," IEEE Trans. Computers, vol. 40, no. 1, pp. 2-12, 1991.

[16] P. M. Merlin and P. J. Schweitzer, "Deadlock avoidance in store-and-forward networks - I: store-and-forward deadlock," IEEE Trans. Communications, vol. 28, no. 3, pp. 345-354, 1980.

[17] J. Y. Ngai and C. L. Seitz, "A framework for adaptive routing in multicomputer networks," Computer Architecture News, vol. 19, no. 1, pp. 6-14, March 1991.

[18] L. M. Ni and P. K. McKinley, "A survey of wormhole routing techniques in direct networks," IEEE Computer, vol. 26 , no. 2, pp. 62-76, 1993.

[19] E. M. Reingold, J. Nievergelt and N. Deo, Combinatorial Algorithms, Prentice-Hall, Englewood Cliffs, NJ, 1977.

[20] R. Suaya and G. Birtwistle, ed., VLSI and Parallel Computation, Morgan Kaufmann Publishers Inc., San Mateo, CA, 1990.

\section{Appendix A}

\section{Procedure DRAW-P-FORM}

Input: a digraph $G=(V, C)$

Output: a P-form of $G$ and the associated level value $l(u)$ for all $u \in V$

\section{Begin}

Choose arbitrarily a node $r$ as the root; $l(r) \leftarrow 0$;

Draw $r$ at the bottom;

Mark each node in $G$ as unvisited; Mark $r$ as visited;

$i \leftarrow 0$;

Repeat $\quad 1^{*}$ Level $i+1$ is located one layer for each unvisited node $v \quad$ above level $i * /$

if $\langle u, v\rangle \in C, l(u) \leq i, u$ is visited and $(\forall w, l(w)=i+1,<w, v>\notin C)$

then begin

Mark $v$ as visited; $l(v) \leftarrow i+1$;

Draw $v$ at the $(i+1)$-th level;

Draw all directed edges $\langle p, v>\in C$ and $\langle\nu, p>\in C$, where $\langle p, v>\in C$ and $l(p) \leq i$

end;

$i \leftarrow i+1$

End 


\section{Appendix B}

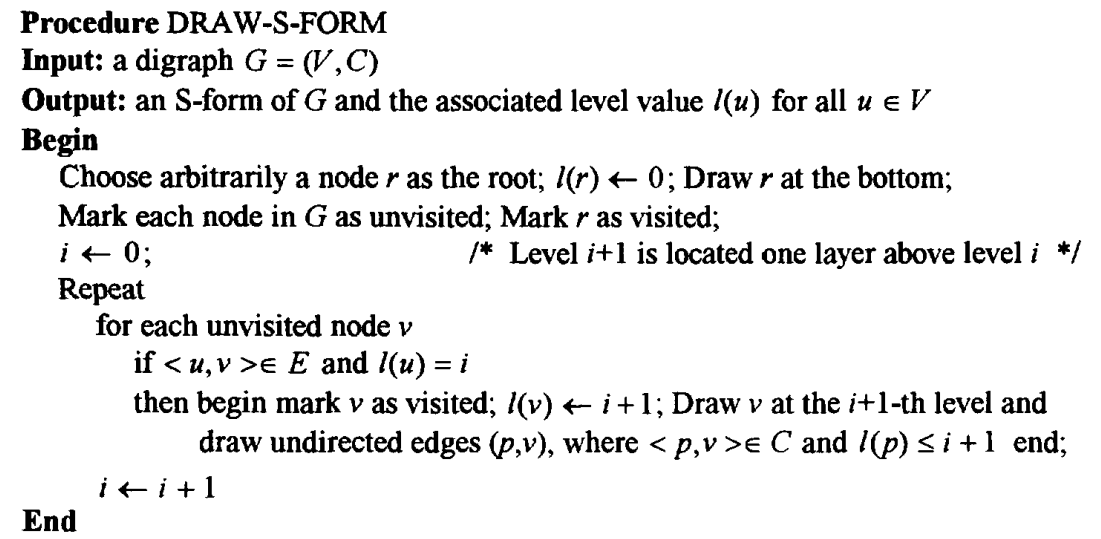

\section{Appendix C}

Algorithm HP-ROUTE

/* Current node $u$ receives a header flit $(v, i)$, where $v$ is destination and $i$ is routing information */

$1 *$ The routing information is 1 if previous channel used is a downward channel $* /$

/* The routing information is 2 if previous channel used is an upward channel */

/* If node $u$ is the source node of the message, the header flit has $(v, 1) * /$

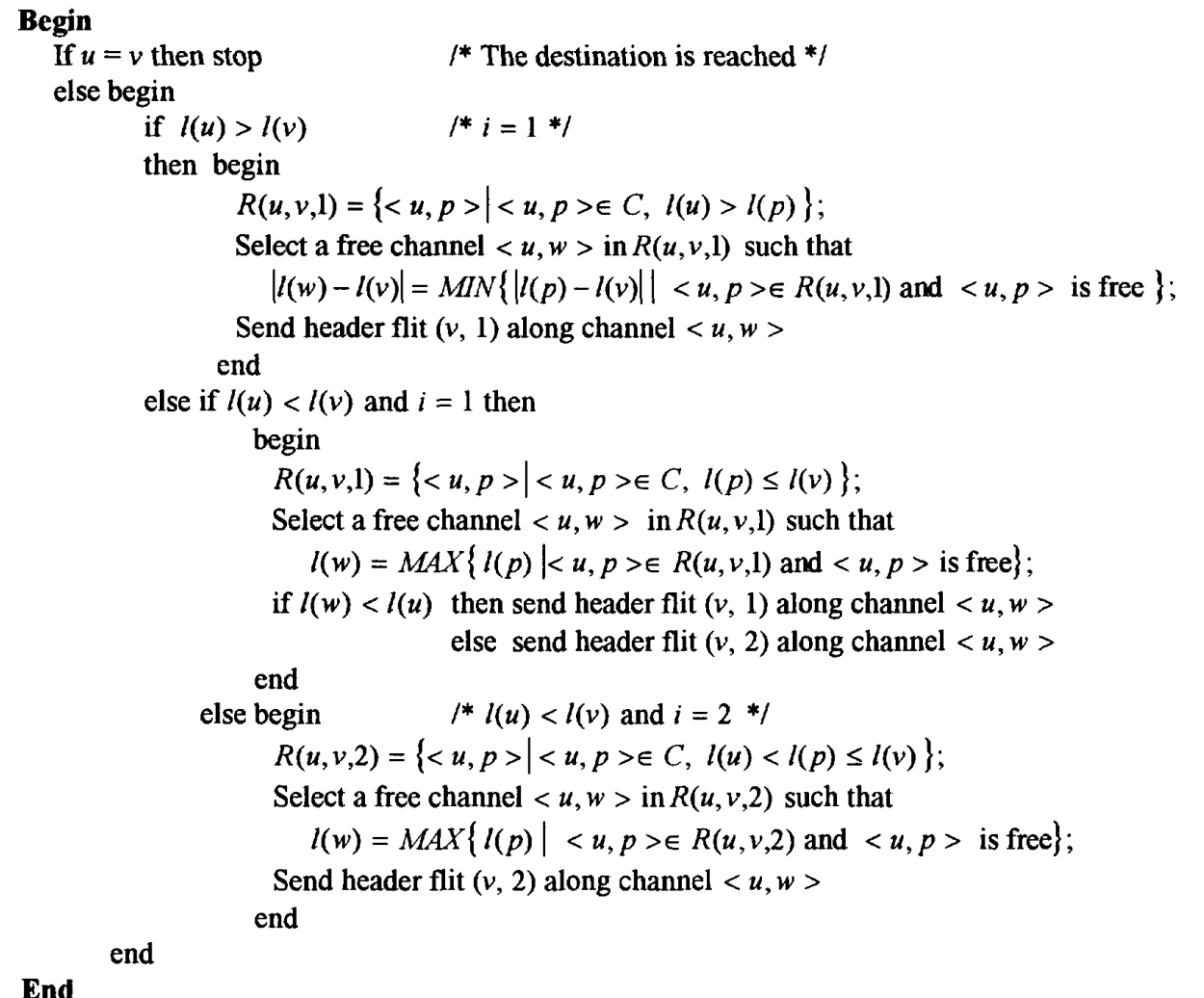

